\title{
Erratum to: Effect of Therapeutic Interventions on Health-related Quality of Life in Parkinson's Disease
}

Heinz Reichmann, ${ }^{1}$ Pablo Martínez-Martin² and Fabrizio Stocchi ${ }^{3}$

1. Professor of Neurology and Chairman, Department of Neurology, University of Dresden Medical School, Dresden, Germany;

2. Neurologist, Tenured Scientist of the Public Boards of Research, and Head of the Neuroepidemiology Section, National Centre of Epidemiology and CIBERNED, Carlos III Institute of Health, Madrid, Spain; 3. Professor of Neurology and Director, Parkinson's Disease and Movement Disorders Research Centre, Institute for Research and Medical Care, IRCCS San Raffaele, Rome, Italy

Erratum to: Heinz Reichmann, Pablo Martínez-Martin and Fabrizio Stocchi. Effect of Therapeutic Interventions on Health-related Quality of Life in Parkinson's Disease. European Neurological Review, 2014;9(1):19-26

The authors of the above-mentioned paper would like to make the following adjustment to their article.

The title of Table 1 on the second page should read "Level of Evidence Supporting Early Parkinson's Disease Drugs Based on Efficacy Against Motor Symptoms".

The authors thank Touch Medical Media for publishing this correction.

Received: 2 September 2014 Citation: European Neurological Review, 2014;9(2):156 DOI:10.17925/ENR.2014.09.02.156

\section{w w w. † o chneurology.com}

\section{SUPPORTING EXCELLENCE IN CLINICAL PRACTICE}

